\title{
Fresh versus frozen-thawed blastocyst transfer in high responders
}

\author{
Ziya Kalem ${ }^{1}$, Muberra Namli Kalem ${ }^{2}$, , Halil Ruso ${ }^{1}$, Batuhan Bakirarar ${ }^{4}$, Timur Gurgan ${ }^{1}$ \\ ${ }^{1}$ Gurgan Clinic IVF and Women Health Center, Ankara, Turkey \\ ${ }^{2}$ Liv Hospital Ankara, Ankara, Turkey \\ ${ }^{3}$ Istinye University, Istanbul, Turkey \\ ${ }^{4}$ Ankara University, Faculty of Medicine, Department of Biostatistics, Ankara, Turkey
}

\begin{abstract}
Objectives: This study aimed to investigate and compare the pregnancy and live birth rates in IVF cycles of frozen-thawed embryo transfers and fresh embryo transfers in a group of women with a high risk of Ovarian hyperstimulation syndrome (OHSS).

Material and methods: The study group consisted of 254 women with a high level of response to controlled ovarian hyperstimulation. The patients who received fresh cycle embryo transfers with calcium infusions are referred to as the Fresh $\mathrm{Ca}+$ group, and those without the calcium therapy are called the Fresh Ca- group; and we used correspondingly similar terminology for the Frozen group.

Results: We observed no statistically significant differences between the cycles of fresh and frozen-thawed embryo transfers in patients with a high risk of OHSS in terms of implantation, clinical pregnancy, and live birth rates. Furthermore, these implantation, clinical pregnancy and live birth rates were not different in the cycles with or without calcium treatment. There was no statistical difference in the OHSS rates between the fresh and frozen-thawed cycles; although, the OHSS rates were less in the two calcium infusion groups (Fresh $\mathrm{Ca}+$ and Frozen-thawed $\mathrm{Ca}+$ ) than in the without-calcium group. There was no OHSS development in the subjects of the Frozen-thawed $\mathrm{Ca}+$ group.

Conclusions: Our study results suggest that fresh and frozen-thawed embryo transfers have similar IVF results in patients with a high risk of OHSS. Calcium infusion is beneficial in preventing OHSS without altering pregnancy rates. Both IVF protocols with calcium infusion can safely be applied in high-responder patients without lowering success rates.
\end{abstract}

Key words: OHSS, high responder, thaw, fresh cycle, blastocyst transfer

\section{INTRODUCTION}

The quality of the embryo, and of endometrial receptivity, and a well-balanced embryo-endometrium interaction are the most important determinants of success in Assisted Reproduction Techniques (ART) [1]. There is evidence that exposure to high levels of estrogen $\left(E_{2}\right)$ and progesterone (P) during controlled ovarian stimulation (COS) leads to biochemical and morphological modifications and can impair endometrial receptivity [2-4]. Such changes in turn lead to an asynchrony between the embryo and the endometrium, which can cause the impairment of the embryo implantation [5]. However, to date, there is no consensus about the thre- shold at which a cycle becomes supraphysiological leading to changes in endometrial receptivity [6].

Frozen embryo transfer (FET) pregnancy rates were higher in studies comparing IVF cycles of both fresh and frozen embryo transfers $[5,7,8]$. These studies show that in transfers of fresh embryos, the endometrial receptivity is impaired because of the high levels of estrogen during controlled ovarian hyperstimulation; and for that reason, fresh embryo transfers may have lower implantation chances with resulting lower pregnancy rates. Comparing the group where the best embryo was obtained for fresh transfer (and the remaining embryos were frozen for a later 
frozen-thawed cycle) with the group of women whose embryos were all frozen before any transfer, the latter group showed better results than the subsequent frozen-thawed cycles for the former group where the best embryo available was only the "second best" [9].

A freeze-all method is also one of the effective ways to eliminate the risk of ovarian hyperstimulation syndrome (OHSS) during the ART cycle [10]. OHSS is one of the major complications of COS; and it is iatrogenic and potentially lethal at present [11].

\section{Objectives}

In this study, we aimed to investigate and compare the pregnancy and live birth rates of two groups of women with a high risk of Ovarian hyperstimulation syndrome (OHSS) who underwent IVF cycles of frozen-thawed embryo transfers and fresh embryo transfers (wherein all the embryos obtained from the fresh cycle were frozen and the embryos were only transferred after thawing, at a later stage, in a subsequent cycle, during which the endometrium was artificially prepared.

\section{MATERIAL AND METHODS}

This is a retrospective study of a total of 254 patients who underwent ICSI in a private IVF center between 2013 and 2016. The study group consisted of women with a high response to controlled ovarian hyperstimulation (high responder). Patients with a total follicle count of 15 or more and/or an estradiol $\left(E_{2}\right)$ value of over $3000 \mathrm{pg} / \mathrm{mL}$ on the day of ovulation induction were considered as high responders and with a high risk of OHSS. Exclusions from our study: patients over 40 years old, male factor, recurrent pregnancy loss stories, uterine pathologies, chronic systemic medical problems, systemic drug use, and agonist-induced.

The patients in our study were divided into four groups: those who received IVF cycles of fresh embryo transfer with calcium infusion (Fresh Ca+ group), those with fresh embryo transfers without calcium therapy (Fresh Ca- group), those who received IVF cycles of frozen-thawed embryo transfers with calcium infusions (Frozen Ca+ group), and those with frozen-thawed embryo transfers without calcium treatment (Frozen Ca- group).

In our study, gonadotropin-releasing hormone (GnRH) agonist and $\mathrm{GnRH}$ antagonist protocols were administered in patients during COS.

During the agonist protocol, $\mathrm{GnRH}$ agonist $0.5 \mathrm{mg} /$ day leuprolide acetate (Lucrin Daily; Abbott, Istanbul, Turkey) was started in the luteal phase of the previous cycle and recLH treatments were added in addition to recFSH, $\mathrm{HMG}$ or recFSH on the second and third days of the cycle. Drugs used in these treatments included recombinant human follicle stimulated hormone (Rec-hFSH); either follitropin-a (Gonal-F ; Ares Serono, Geneva, Switzerland) or follitropin- $\beta$ (Puregon ;
Organon, Oss, the Netherlands); either human menopausal gonadotropin (HMG) (Menogon; Ferring, Istanbul, Turkey) or highly purified human menopausal gonadotropin (HP-HMG) (Menopur; ; Ferring, Istanbul, Turkey); and recLH (Luveris; Merck Serono, Istanbul, Turkey).

In the antagonist protocol, we commenced the gonadotrophins on the second or third day of the cycle, and cetrorelix (Cetrotide ${ }^{\circ}$; Merck Serono, Turkey) was added to the treatment as a $\mathrm{GnRH}$ antagonist, when the dominant follicle was $14 \mathrm{~mm}$.

In both protocols, the development of the follicles was followed by transvaginal USG and E2. When the dominant follicle measurement was $19 \mathrm{~mm}$ or more, or at least three follicles were $17 \mathrm{~mm}$ or more, gonadotropin administration was terminated, and ovulation was induced. Ovulation was induced with $250 \mu \mathrm{g}$ of human recombinant hCG (Ovitrelle Merck Serono, Turkey). Oocyte retrieval was performed during the transvaginal USG process between the $36^{\text {th }}$ and $40^{\text {th }}$ hour after ovulation was induced, and this was followed by intracytoplasmic sperm injection (ICSI) with mature oocytes and fresh sperm.

Our study involved embryo transfers at the fresh cycle for 181 patients; and 73 patients in the frozen-thawed group whose embryos were frozen in total at the ovarian stimulation cycle and then transferred at a later stage. Vitrification was employed to freeze the embryos.

Calcium infusion was performed in 72 patients of the total group and in 28 patients of the total frozen group. Calcium infusion was administered intravenously by adding $10 \%$ calcium gluconate (one $\mathrm{gr} / 10 \mathrm{~mL}$ ) in ampules into the physiologic saline. In addition, calcium infusion was administered as one gram on the day of the hCG induction and as two grams on the oocyte retrieval day. The standard protocol of our clinic is for all patients with a high risk of OHSS, including all the patients in our study to receive $0.5 \mathrm{mg} /$ day cabergoline tablets (Dostinex ${ }^{\circledR}$, Pharmacia, Berlin, Germany) until the day of their embryo transfer when more than $12 \mathrm{fol}-$ licles are observed to be over $14 \mathrm{~mm}$.

In both study groups (fresh and frozen-thawed cycles), we only selected those patients who underwent blastocyst transfer. The preparation of endometrium in all the thaw cycles was performed using transdermal estrogen (Estraderm TTS; Novartis, Istanbul, Turkey). The luteal phase support started on the day of oocyte retrieval. All patients received vaginal micronized progesterone in gel form (Crinone 8\%; Merck Serono, Istanbul, Turkey) in a single daily administration. Progesterone was used until nine weeks, if pregnancy was confirmed.

\section{RESULTS}

A total of 254 high-responder patients were included in our study. Fresh embryo transfers were performed on 
181 patients $(71.2 \%)$, while frozen-thawed embryo transfers were performed on 73 patients (28.7\%). Calcium treatment was administered to 109 patients $(60 \%)$ in the fresh groups; and $45(61.6 \%)$ of these patients received the calcium treatment as part of their frozen-thawed transfer. Table 1 shows the patients' ages, body mass index (BMI), hormonal values at the beginning of the IVF cycle, infertility duration and which IVF cycle.

The results of our transvaginal USG and ovarian evaluation of the study patients show that 149 (58\%) of them had a normofollicular appearance, and 105 (41.3\%) of them had a polycystic ovary (PCO) appearance. Among the patients who appeared to have PCO, 84 (80\%) were diagnosed with polycystic ovary syndrome (PCOS) and 21 (20\%) were diagnosed with PCO. The menstruation cycles were regular in $172(67.7 \%)$ of our patients, whereas they were irregular in $82(32.2 \%)$ of the patients. 221 patients $(87 \%)$ were diagnosed with primary infertility, whereas 33 (12.9\%) were diagnosed with secondary infertility. 15 (5.9\%) patients had a previous OHSS history. In addition, one had had OHSS twice and another patient had had OHSS three times.

Considering the COS protocols used in the study: in the fresh cycles, we used the antagonist protocol with 121 patients (66.8\%) and the long-agonist protocol with 60 (33.1\%); and in the frozen cycles we used the antagonist protocol with $60(82.1 \%)$ of the patients and the long-agonist protocol with 13 patients (17.8\%).

In the study's administration of drugs, 142 patients (79.0\%) received $\mathrm{FSH}, 16$ patients (8.8\%) received HMG, 12 patients $(6.6 \%)$ received $\mathrm{FSH}+\mathrm{HMG}$, and 10 patients (5.5\%) received $\mathrm{FSH}+\mathrm{LH}$. Of the patients in the frozen groups, 58 (79.4\%) received $\mathrm{FSH}, 11$ (15\%) received HMG, one (1.3\%) received $\mathrm{FSH}+\mathrm{HMG}$, and three $(4.1 \%)$ received $\mathrm{FSH}+\mathrm{LH}$.

In the ovulation induction process, of the fresh groups, 138 patients (76.2\%) received rec-hCG and 43 patients $(23.7 \%)$ received urinary $\mathrm{hCG}$. Of the patients in the frozen groups, 46 (63.0\%) received rec-hCG and 27 (36.9\%) received u-hCG.

Table 2 shows the comparative characteristics of the cycle for the four study groups. Table 3 shows the comparative results of the cycle for the four groups. There were no statistically significant differences between the groups for implantation rates, clinical pregnancy rates, and live birth rates.

The study showed the OHSS development rates across the groups as follows: 2 individuals (1.8\%) in the Fresh $\mathrm{Ca}+$ group, $6(8.3 \%)$ in the Fresh Ca- group, none $(0 \%)$ in the Frozen $\mathrm{Ca}+$ group, and 1 (3.6\%) in the Frozen Ca- group. There was no statistically significant correlation between the groups' rates of OHSS development $(p=0.065)$. When the OHSS development rates of the 2 frozen groups were compared with those of the two fresh groups, development occurred in 8 patients (4.4\%) of the combined frozen groups, and in 1 (1.4\%) of the combined fresh groups. There was no statistically significant correlation between the results for the fresh and frozen groups' rates of OHSS development ( $p=0.453$ ). When the OHSS development rates of those patients who received calcium was compared with those who did not receive calcium, OHSS developed in 2 (1.3\%) of the former, and in 7 (7.0\%) of the latter group that did not receive calcium treatment. There was a statistically significant difference between the OHSS development results for these two with- and without-calcium ( $p=0.031$ ).

In the Fresh $\mathrm{Ca}+$ group, early OHSS developed in 1 (50.0\%) patient, and late OHSS developed in 1 (50.0\%) other. In the Fresh Ca- group, 2 (33.3\%) of the 6 patients were early OHSS, whereas 4 (66.7\%) of the 6 patients were late OHSS. In the Frozen Ca- group, early OHSS developed in one $(100.0 \%)$ patient.

Comparing the patients who developed OHSS, 4 (44.4\%) had mild OHSS, 4 (44.4\%) had moderate OHSS, and 1 (11.2\%) had severe OHSS. Distribution of these individuals across the study groups was as follows: one patient with mild OHSS and one with moderate OHSS in the Fresh Ca+ group; three patients with mild OHSS, two with moderate OHSS, and one with severe OHSS in the Fresh Ca- group; and one patient with moderate OHSS in the Frozen Ca-.

Three (33.3\%) of the nine patients developing OHSS required hospitalization. Two (66.7\%) of those patients were in the Fresh Ca- group, while 1 (33.3\%) was in the Frozen Ca- group.

Two (22.2\%) patients with OHSS had abdominal ascites. One (50.0\%) of these patients was in the Fresh Ca- group, and the other 1 (50.0\%) was in the Frozen Ca- group.

\section{DISCUSSION}

Our study showed no statistically significant differences in the rates of implantation, clinical pregnancies, and live births between the fresh and frozen-thawed cycles in patients with a high risk of OHSS. Furthermore, these rates of implantation, clinical pregnancies, and live births were not differentiated when comparing the cycles with or without calcium treatment. The OHSS development rates were not statistically different when comparing the fresh and thawed cycles; however, when comparing the $\mathrm{Ca}+$ and $\mathrm{Ca}$ - groups, the rate of OHSS development was significantly less in the former. OHSS did not develop in the members of the total freeze group with Ca infusion.

Previous studies have found that supraphysiological estrogen levels had detrimental effects on oogenesis, embryo development, endometrial receptivity and implantation rates in patients with an over-response to $\operatorname{COS}$ [12-14]. In the literature, the frozen-thawed embryo transfer was presented as a good option to protect embryos from endometrium 


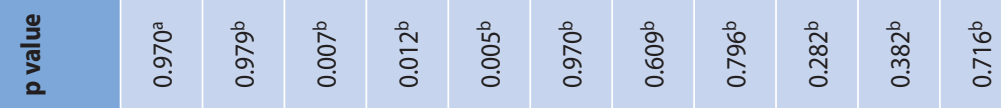

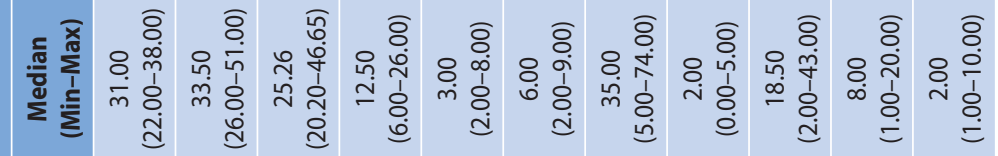
s)

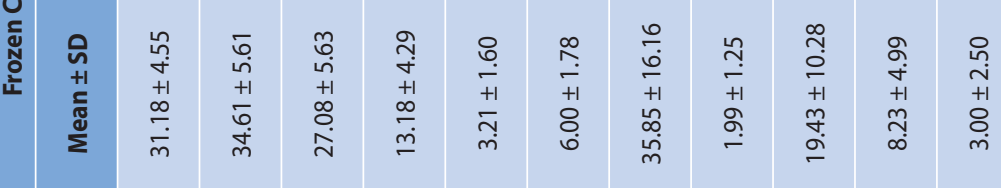

$z \quad \stackrel{\infty}{\sim} \quad \stackrel{\infty}{\sim} \quad \stackrel{\infty}{\sim} \stackrel{\infty}{\sim} \stackrel{\infty}{\sim}$ ฟ $\stackrel{\infty}{\sim} \stackrel{\infty}{\sim}$ ฟ

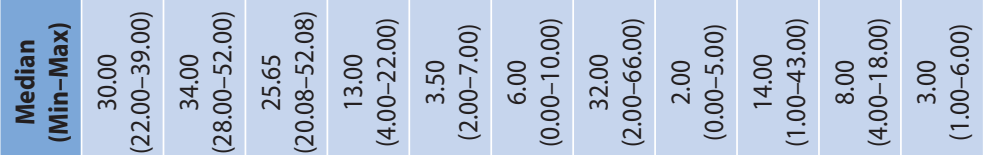
志

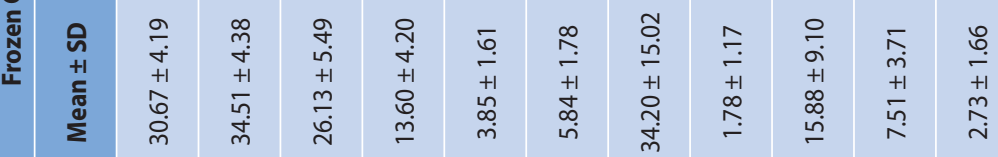

은

z

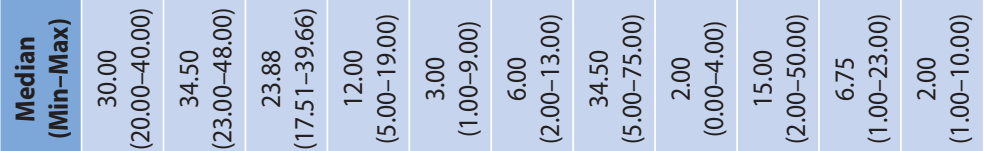

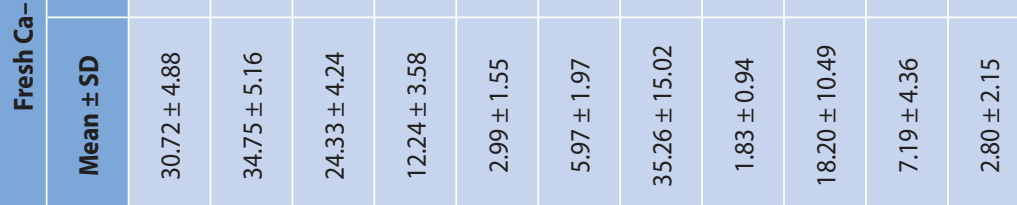

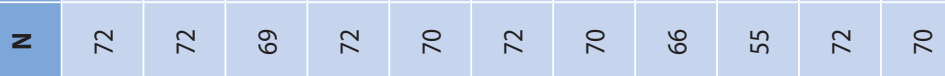

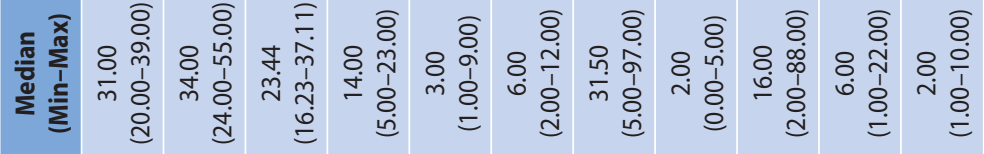
芯

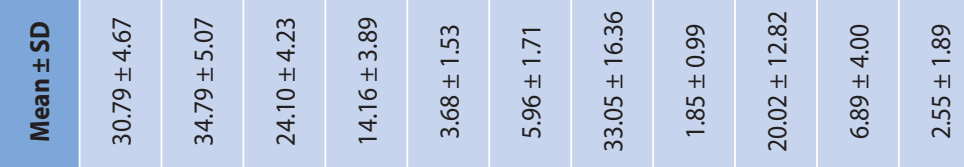

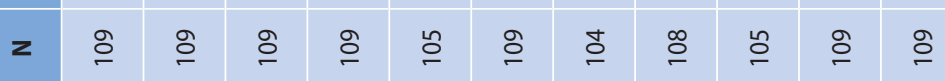

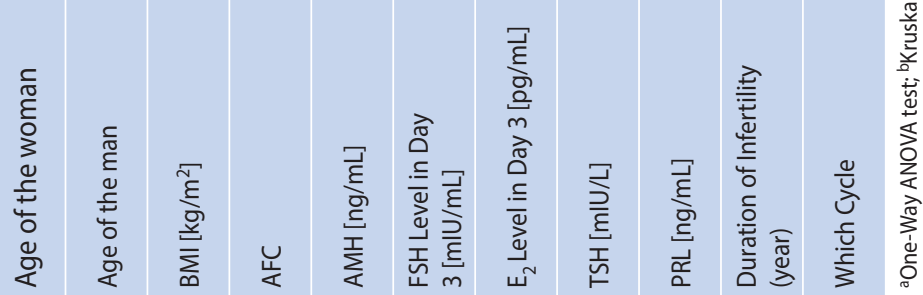




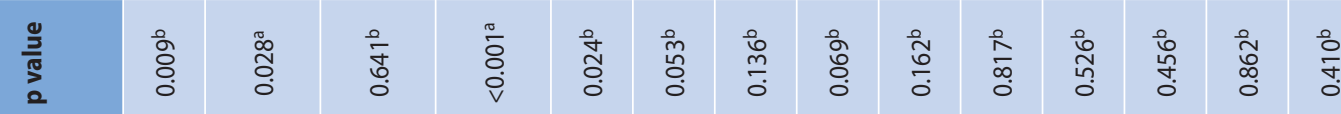

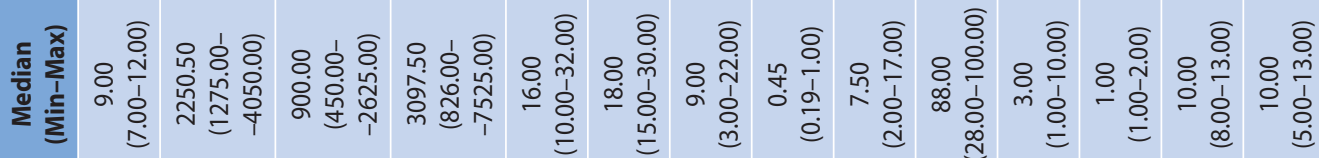
ป⿱

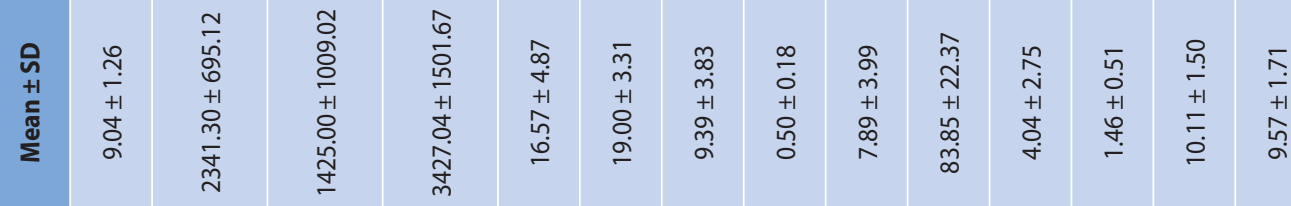

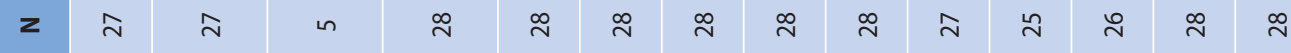

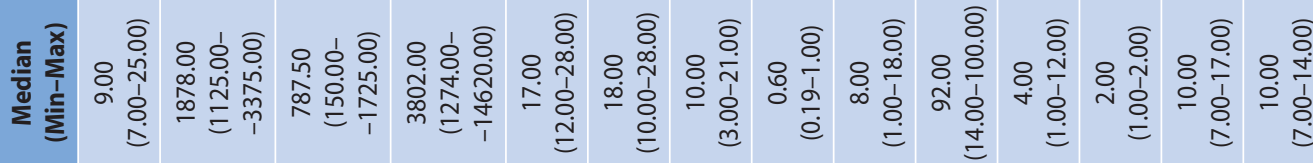
志

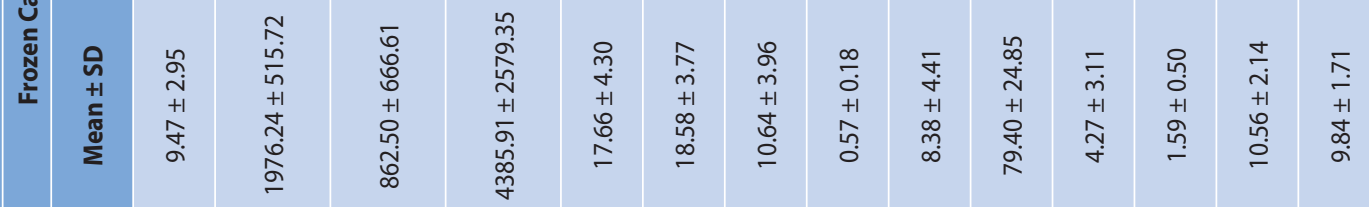

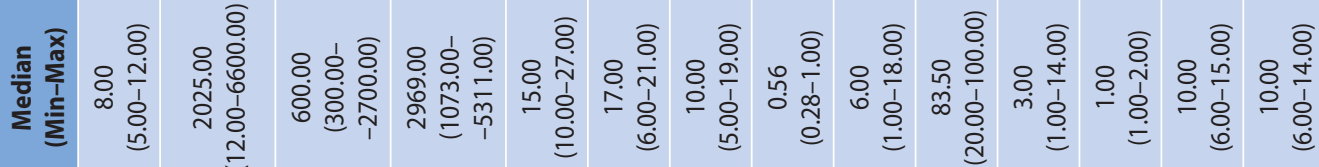

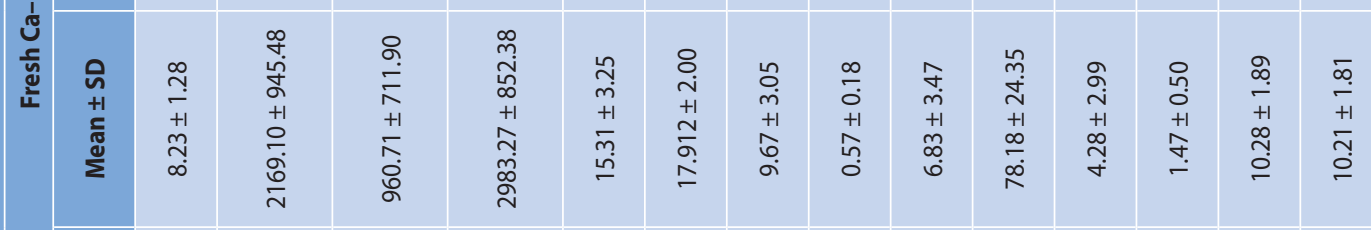

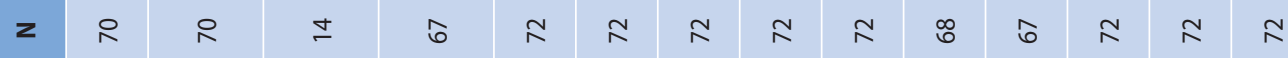

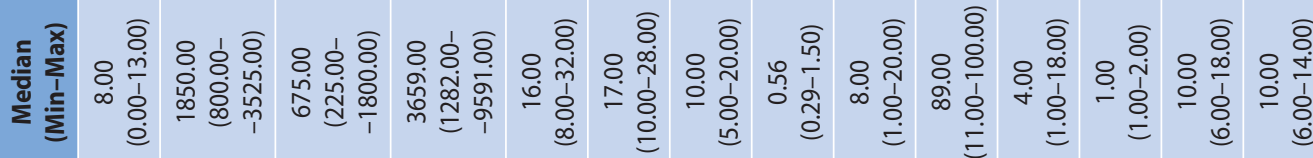

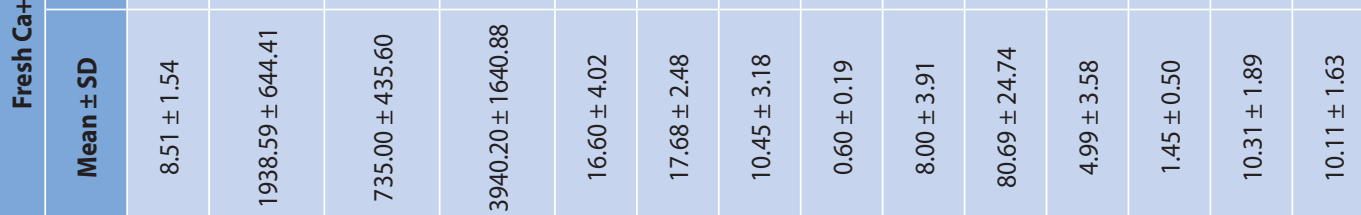

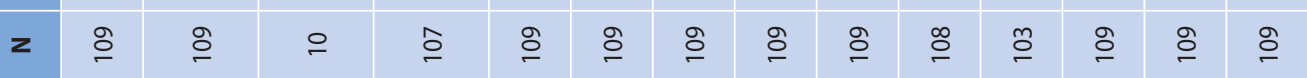

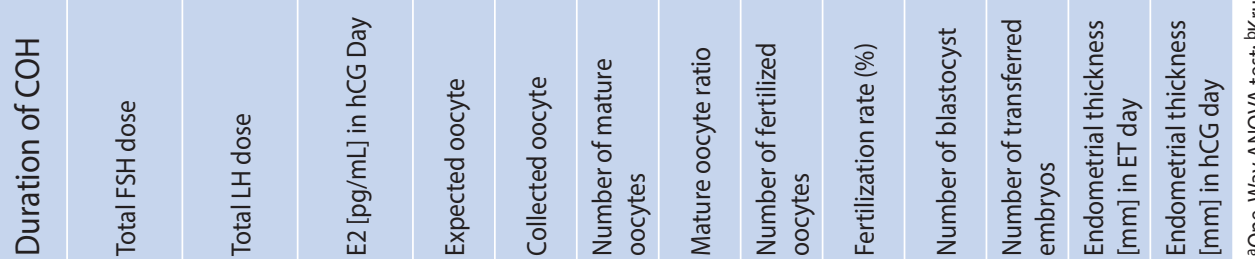


Table 3. Comparison of pregnancy results for the fresh and frozen-thawed embryo transfer groups, with and without calcium treatment

\begin{tabular}{|l|c|c|c|c|c|}
\hline \multirow{2}{*}{ Variables } & \multicolumn{4}{|c|}{ Group } \\
\cline { 2 - 5 } & Fresh Ca+ & Fresh Ca- & Frozen Ca+ & Frozen Ca- \\
\hline Implantation rate per cycle, $\mathrm{n}(\%)$ & $49(45.0)$ & $31(43.1)$ & $21(46.7)$ & $13(46.4)$ \\
\hline Implantation rate per ET, $\mathrm{n}(\%)$ & $49(31.0)$ & $31(29.2)$ & $21(30.0)$ & $13(34.2)$ \\
\hline Clinical pregnancy rate per cycle, $\mathrm{n}(\%)$ & $46(42.2)$ & $28(38.9)$ & $18(40.0)$ & $11(39.2)$ \\
\hline Clinical pregnancy rate per ET, $\mathrm{n}(\%)$ & $46(29.1)$ & $28(26.4)$ & $18(25.7)$ & $11(28.9)$ \\
\hline Live birth rate per cycle, $\mathrm{n}(\%)$ & $46(42.2)$ & $26(36.1)$ & $16(35.6)$ & 9.951 \\
\hline Live birth rate per $\mathrm{ET}, \mathrm{n}(\%)$ & $46(29.1)$ & $26(24.5)$ & $16(22.9)$ & 9.938 \\
\hline
\end{tabular}

exposed to high estrogen $[9,15]$. At the same time, a freeze-all strategy has become an extremely popular method of protecting against OHSS [16].

Comparisons of fresh and frozen-thawed cycles displayed different results. The methodologies, and therefore the data, of four large randomized controlled studies we reviewed and discuss in this paragraph were quite different from each other. Chen et al. [17] reported higher live birth rates following frozen embryo transfers in PCOS patients than in patients from the fresh group. Shapiro et al. [7] reported that implantation rates in normoresponder frozen thawed cycles were higher than both the clinical and ongoing pregnancy rates from fresh cycles, and they attributed these results to the impaired endometrial receptivity of the fresh cycles. Shapiro et al. [18] found no difference between clinical and ongoing pregnancy rates in high responder patients. Ferraretti et al. [19] examined the difference between fresh and frozen-thawed transfers in patients with a risk of OHSS and did not find any differences between the resulting pregnancies and live birth rates for either group.

Our study was conducted with only high responder subjects with a high risk of OHSS who underwent cycles of blastocyst transfer with fresh and frozen-thawed embryos, and there was no statistically significant difference in pregnancy rates between those fresh and frozen-thawed groups. The reason why only included patients who had undergone blastocyst transfers in our study was to homogenize the study group. Discussions in the literature on the relative effects of blastocyst versus cleavage-stage embryo transfers on the maternal and neonatal outcomes are ongoing, as are discussions on the effects on implantation and pregnancy rates [20-23].

Gurgan et al. [24] published a study in 2011 demonstrating that calcium infusion reduced the risk of developing OHSS. In the following years, more studies supporting this finding were published $[25,26]$. Calcium infusion is used for OHSS prevention in the group with high OHSS risk in our clinic. In our study, along with comparing fresh and frozen- -thawed cycles, we compared the pregnancy rates of the groups with and without calcium and it was found that there was no significant difference in the pregnancy rates. Though the risk of OHSS development was significantly reduced in the group receiving calcium treatment, we did observe early and moderate OHSS in this group, along with the need for hospitalization, and abdominal ascites; however, severe OHSS did not develop. Khayat et al. [25] found no difference in implantation and pregnancy rates between groups using and not using calcium, whereas Gurgan et al. [24] reported that pregnancy rates were higher in the calcium-treated group than in the group not receiving calcium.

One of the most successful methods of protecting against OHSS is the use of agonist induction [27]. There are contrasting conclusions in the literature on the topic of the effects of agonist induction versus hCG induction on implantation and pregnancy rates $[28,29]$. Our study did not include an agonist induction group. Since the primary outcome of our study was to compare pregnancy rates resulting from groups of fresh and frozen-thawed cycles, instead of OHSS development rates, only hCG induction cycles were included in the study. In this way the effects of agonist induction on the pregnancy rates of our subjects were eliminated. By the same logic, our study did not include cleavage-stage embryo transfers; therefore, any difference in pregnancy rates between cleavage-stage and blastocyst was also excluded.

Our study is the first involving subjects with a high risk of OHSS who underwent cycles of blastocyst transfer to compare the results of fresh and frozen-thawed embryo transfers. In this respect, we believe that our study will contribute to the literature. The main limitation of our study is its retrospective nature.

In conclusion, our study results suggest that fresh and frozen-thawed embryo transfers have similar IVF results among patients with a high risk of OHSS. Calcium infusion is beneficial in preventing OHSS without altering pregnancy rates. Both calcium infusion protocols can be safely applied in high-responder patients without lowering success rates. 


\section{Compliance with Ethical Standards Funding}

This study did not receive any funding.

Conflict of interest

All author declare that they have no conflict of interest.

Ethical approval

All procedures performed in the studies involving human participants were in accordance with the ethical standards of our institutional ethics committee and with the 1964 Declaration of Helsinki (DoH) and its later amendments. Informed consent

A written consent form was obtained from each patient

\section{REFERENCES}

1. Achache H, Revel A. Endometrial receptivity markers, the journey to successful embryo implantation. Hum Reprod Update. 2006; 12(6): 731-746, doi: 10.1093/humupd/dml004, indexed in Pubmed: 16982667.

2. Groothuis PG, Dassen HH, Romano A, et al. Estrogen and the endometrium: lessons learned from gene expression profiling in rodents and human. Hum Reprod Update. 2007; 13(4): 405-417, doi: 10.1093/humupd/dmm009, indexed in Pubmed: 17584823.

3. Ullah K, Rahman TUr, Pan HT, et al. Serum estradiol levels in controlled ovarian stimulation directly affect the endometrium. J Mol Endocrinol. 2017; 59(2): 105-119, doi: 10.1530/JME-17-0036, indexed in Pubmed: 28539318.

4. Venetis CA, Kolibianakis EM, Papanikolaou E, et al. Is progesterone elevation on the day of human chorionic gonadotrophin administration associated with the probability of pregnancy in in vitro fertilization? A systematic review and meta-analysis. Hum Reprod Update. 2007; 13(4): 343-355, doi: 10.1093/humupd/dmm007, indexed in Pubmed: 17405832.

5. Roque $M$, Lattes $K$, Serra $S$, et al. Fresh embryo transfer versus frozen embryo transfer in in vitro fertilization cycles: a systematic review and meta-analysis. Fertil Steril. 2013; 99(1): 156-162, doi: 10.1016/j.fertnstert.2012.09.003, indexed in Pubmed: 23040524.

6. Barnhart KT. Introduction: are we ready to eliminate the transfer of fresh embryos in in vitro fertilization? Fertil Steril. 2014; 102(1): 1-2, doi: 10.1016/j.fertnstert.2014.05.024, indexed in Pubmed: 24890272.

7. Shapiro BS, Daneshmand ST, Garner FC, et al. Evidence of impaired endometrial receptivity after ovarian stimulation for in vitro fertilization: a prospective randomized trial comparing fresh and frozen-thawed embryo transfer in normal responders. Fertil Steril. 2011; 96(2): 344-348, doi: 10.1016/j.fertnstert.2011.05.050, indexed in Pubmed: 21737072.

8. Roque $M$, Valle $M$, Guimarães $F$, et al. Freeze-all policy: fresh vs. frozen-thawed embryo transfer. Fertil Steril. 2015; 103(5): 1190-1193, doi: 10.1016/j.fertnstert.2015.01.045, indexed in Pubmed: 25747130.

9. Shapiro BS, Daneshmand ST, Garner FC, et al. Clinical rationale for cryopreservation of entire embryo cohorts in lieu of fresh transfer. Fertil Steril. 2014; 102(1): 3-9, doi: 10.1016/j.fertnstert.2014.04.018, indexed in Pubmed: 24842675.

10. Griesinger G, Schultz L, BauerT, et al. Ovarian hyperstimulation syndrome prevention by gonadotropin-releasing hormone agonist triggering of final oocyte maturation in a gonadotropin-releasing hormone antagonist protocol in combination with a "freeze-all"strategy: a prospective multicentric study. Fertil Steril. 2011; 95(6): 2029-33, 2033.e1, doi: 10.1016/j. fertnstert.2011.01.163, indexed in Pubmed: 21371705.

11. Mouzon Jde, Goossens V, Bhattacharya S, et al. Assisted reproductive technology in Europe, 2007: results generated from European registers by ESHRE. Human Reproduction. 2012; 27(4): 954-966, doi: 10.1093/humrep/des023.

12. Santos MA, Kuijk EW, Macklon NS. The impact of ovarian stimulation for IVF on the developing embryo. Reproduction. 2010; 139(1): 23-34, doi: 10.1530/REP-09-0187, indexed in Pubmed: 19710204.
13. Horcajadas JA, Riesewijk A, Polman J, et al. Effect of controlled ovarian hyperstimulation in IVF on endometrial gene expression profiles. Mol Hum Reprod. 2005; 11(3): 195-205, doi: 10.1093/molehr/gah150, indexed in Pubmed: 15695772

14. Zhu L, LiY, Xu A. Influence of controlled ovarian hyperstimulation on uterine peristalsis in infertile women. Hum Reprod. 2012; 27(9): 2684-2689, doi: 10.1093/humrep/des257, indexed in Pubmed: 22798632.

15. Weinerman R, Mainigi M. Why we should transfer frozen instead of fresh embryos: the translational rationale. Fertil Steril. 2014; 102(1): 10-18, doi: 10.1016/j.fertnstert.2014.05.019, indexed in Pubmed: 24890274.

16. Roque $M$, Valle $M$, Guimarães $F$, et al. Freeze-all policy: fresh vs. frozen-thawed embryo transfer. Fertil Steril. 2015; 103(5): 1190-1193, doi: 10.1016/j.fertnstert.2015.01.045, indexed in Pubmed: 25747130.

17. Chen ZJ, Shi Y, Sun Y, et al. Fresh versus frozen embryos for infertility in the polycystic ovary syndrome. N Engl J Med. 2016; 375(6): 523-533, doi: 10.1056/NEJMoa1513873, indexed in Pubmed: 27509101.

18. Shapiro BS, Daneshmand ST, Garner FC, et al. Evidence of impaired endometrial receptivity after ovarian stimulation for in vitro fertilization: a prospective randomized trial comparing fresh and frozen-thawed embryo transfers in high responders. Fertil Steril. 2011; 96(2): 516-518, doi: 10.1016/j.fertnstert.2011.02.059, indexed in Pubmed: 21737071.

19. Ferraretti AP, Gianaroli L, Magli C, et al. Elective cryopreservation of all pronucleate embryos in women at risk of ovarian hyperstimulation syndrome: efficiency and safety. Human Reproduction. 1999; 14(6): 1457-1460, doi: 10.1093/humrep/14.6.1457.

20. Martins WP, Nastri CO, Rienzi L, et al. Blastocyst vs cleavage-stage embryo transfer: systematic review and meta-analysis of reproductive outcomes. Ultrasound Obstet Gynecol. 2017; 49(5): 583-591, doi: 10.1002/uog.17327, indexed in Pubmed: 27731533.

21. Maheshwari A, Hamilton M, Bhattacharya S. Should we be promoting embryo transfer at blastocyst stage? Reprod Biomed Online. 2016; 32(2): 142-146, doi: 10.1016/j.rbmo.2015.09.016, indexed in Pubmed: 26673100.

22. De Vos A, Van Landuyt L, Santos-Ribeiro S, et al. Cumulative live birth rates after fresh and vitrified cleavage-stage versus blastocyst-stage embryo transfer in the first treatment cycle. Hum Reprod. 2016; 31(11): 2442-2449, doi: 10.1093/humrep/dew219, indexed in Pubmed: 27619768.

23. Källén B, Finnström $O$, Lindam $A$, et al. Blastocyst versus cleavage stage transfer in in vitro fertilization: differences in neonatal outcome? Fertil Steril. 2010; 94(5): 1680-1683, doi: 10.1016/j.fertnstert.2009.12.027, indexed in Pubmed: 20137785.

24. Gurgan T, Demirol A, Guven S, et al. Intravenous calcium infusion as a novel preventive therapy of ovarian hyperstimulation syndrome for patients with polycystic ovarian syndrome. Fertil Steril. 2011; 96(1): 53-57, doi: 10.1016/j.fertnstert.2011.04.094, indexed in Pubmed: 21621772.

25. El-Khayat W, Elsadek M. Calcium infusion for the prevention of ovarian hyperstimulation syndrome: a double-blind randomized controlled trial. Fertil Steril. 2015; 103(1): 101-105, doi: 10.1016/j.fertnstert.2014.09.046, indexed in Pubmed: 25552412.

26. Naredi N, Karunakaran S. Calcium gluconate infusion is as effective as the vascular endothelial growth factor antagonist cabergoline for the prevention of ovarian hyperstimulation syndrome. J Hum Reprod Sci. 2013; 6(4): 248-252, doi: 10.4103/0974-1208.126293, indexed in Pubmed: 24672164.

27. Devroey P, Polyzos NP, Blockeel C. An OHSS-Free Clinic by segmentation of IVF treatment. Hum Reprod. 2011; 26(10): 2593-2597, doi: 10.1093/humrep/der251, indexed in Pubmed: 21828116.

28. Kolibianakis EM, Schultze-Mosgau A, Schroer A, et al. A lower ongoing pregnancy rate can be expected when $\mathrm{GnRH}$ agonist is used for triggering final oocyte maturation instead of HCG in patients undergoing IVF with GnRH antagonists. Hum Reprod. 2005; 20(10): 2887-2892, doi: 10.1093/humrep/dei150, indexed in Pubmed: 15979994.

29. Acevedo B, Gomez-Palomares JL, Ricciarelli E, et al. Triggering ovulation with gonadotropin-releasing hormone agonists does not compromise embryo implantation rates. Fertil Steril. 2006; 86(6): 1682-1687, doi: 10.1016/j.fertnstert.2006.05.049, indexed in Pubmed: 17074344. 